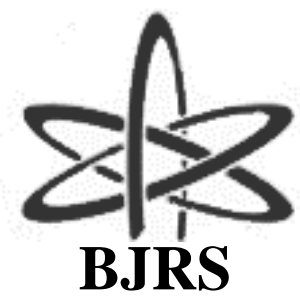

BRAZILIAN JOURNAL

$\mathrm{OF}$

RADIATION SCIENCES

03-1A (2015) 01-09

\title{
Testes preliminares em um simulador pediátrico de crânio para dosimetria em tomografia computadorizada
}

\author{
E. W. Martins; M. P. A. Potiens \\ Gerência de Metrologia das Radiações, Laboratório de Calibração de Instrumentos, \\ Instituto de Pesquisas Energéticas e Nucleares, CEP: 05508-000, \\ São Paulo-SP, Brasil \\ elainewirney@usp.br
}

\begin{abstract}
RESUMO
A tomografia computadorizada (TC) é uma das técnicas na área da radiologia com avanço tecnológico marcante nos últimos anos. Um dos fatores para resultar em tal avanço foi devido ao aumento de número de canais associado ao aumento da potência do tubo de raios X. Essas condições permitiram ao equipamento maior velocidade na aquisição dos cortes, reduzindo assim o tempo de exposição do paciente, característica fundamental para o acréscimo de seu uso em pacientes pediátricos. Neste contexto, foi desenvolvido um novo simulador de crânio pediátrico para análise dos resultados de medições realizadas em feixes laboratoriais e clínicos com o propósito de criação e utilização de níveis de referência de diagnóstico observando riscos dos efeitos estocásticos, além de inevitavelmente avaliar à redução de doses absorvidas em pacientes em fase de desenvolvimento. Testes preliminares realizados em feixes clínicos demonstraram valores de $C_{w}: 2,525 \pm 0,212$ mGy para o simulador desenvolvido citado e 3,362 $\pm 0,282$ mGy para um simulador desenvolvido pelo IPEN denominado de padrão, ambos estando entre os valores de incerteza de $8,4 \%$ e $14,4 \%$ sugerido pela TRS. $n^{\circ}$. 457.
\end{abstract}

Palavras-chave: radiodiagnóstico, tomografia computadorizada, simulador pediátrico

\section{INTRODUÇÃO}

A tomografia computadorizada (TC) é uma das técnicas na área da radiologia com avanço tecnológico marcante nos últimos anos. Um dos fatores responsáveis por resultar nesse avanço foi 
devido ao aumento de número de canais associado ao aumento da potência do tubo de raios X. Essas condições permitiram ao equipamento maior velocidade na aquisição dos cortes, reduzindo assim o tempo de exposição do paciente, embora os parâmetros técnicos utilizados em procedimentos realizados em pacientes adultos devam ser alterados em protocolos pediátricos.

As crianças e os jovens apresentam estruturas corporais menores quando comparada aos adultos, além do que estão em fase de crescimento, tornando-se mais suscetíveis ao desenvolvimento de câncer no decorrer de suas vidas. Assim a redução do tempo para a realização do exame (tecnologia subsecond) é sem dúvida um dos principais motivos para os maiores aumentos no uso da TC para a categoria de diagnóstico pediátrico, permitindo, na maioria das vezes, a eliminação do uso de anestesia para evitar que a criança se mova durante a aquisição da imagem [1].

É frequente a preocupação com os pacientes pediátricos e jovens submetidos a exames realizados em TC devido a ficarem expostos às altas doses de radiação, mesmo que essa técnica forneça eficiência em diagnosticar patologias com precisão. Embora a International Atomic Energy Agency (IAEA) tenha publicado no ano de 2011 a implementação do Technical reports series $n^{\circ}$. 457 (2007) visando diminuir a incerteza na dosimetria de feixes de radiodiagnósticos ainda assim percebeu a necessidade de orientação sobre as normas de dosimetria e metodologias relacionadas com a dosimetria para pacientes pediátricos e em dezembro de 2013 publicou a série 24 - Dosimetria em Radiologia Diagnóstica para Pacientes Pediátricos [2, 3, 4].

As grandezas dosimétricas utilizadas para dosimetria em CT são: índice de kerma no ar livre $\left(\mathrm{C}_{\mathrm{a}, 100}\right)$, índice ponderado de dose $\left(\mathrm{C}_{\mathrm{w}}\right)$, média do índice de kerma no ar volumétrico $\left(\mathrm{C}_{\mathrm{vol}}\right)$, e o produto de Kar-comprimento $\left(\mathrm{P}_{\mathrm{KL}}\right)$. Para determinar ou avaliar essas grandezas é necessário a utilização de simuladores físicos ou computacionais específicos para esse fim.

Os simuladores utilizados no Brasil para a realização da medição do kerma no ar são aqueles recomendados pela Portaria 453 do Ministério da Saúde, na qual foram estabelecidos também os níveis de referência de diagnósticos em CT, contudo para paciente adulto típico [5] Pensando nisso foi desenvolvido no Laboratório de Calibração de Instrumentos (LCI) pertencente ao Instituto de Pesquisas Energéticas e Nucleares (IPEN) um simulador pediátrico com dimensões aproximadas da cabeça de uma criança de cinco anos de idade.

Neste contexto e adotando metodologias adequadas, o objetivo do presente trabalho foi analisar os resultados dos testes preliminares com o simulador desenvolvido mediante as medições de 
kerma de entrada na superfície e futuramente a criação e utilização de níveis de referência de diagnóstico observando os efeitos estocásticos, com intuito de redução dose em pacientes pediátricos.

\section{MATERIAIS E MÉTODOS}

Todas as medições foram realizadas utilizando uma câmara de ionização do tipo lápis modelo 10X5-3CT acoplada ao eletrômetro modelo 9010, ambos da marca Radcal conforme Figura 1.

Figura 1: Câmara de ionização e eletrômetro (Radcal)

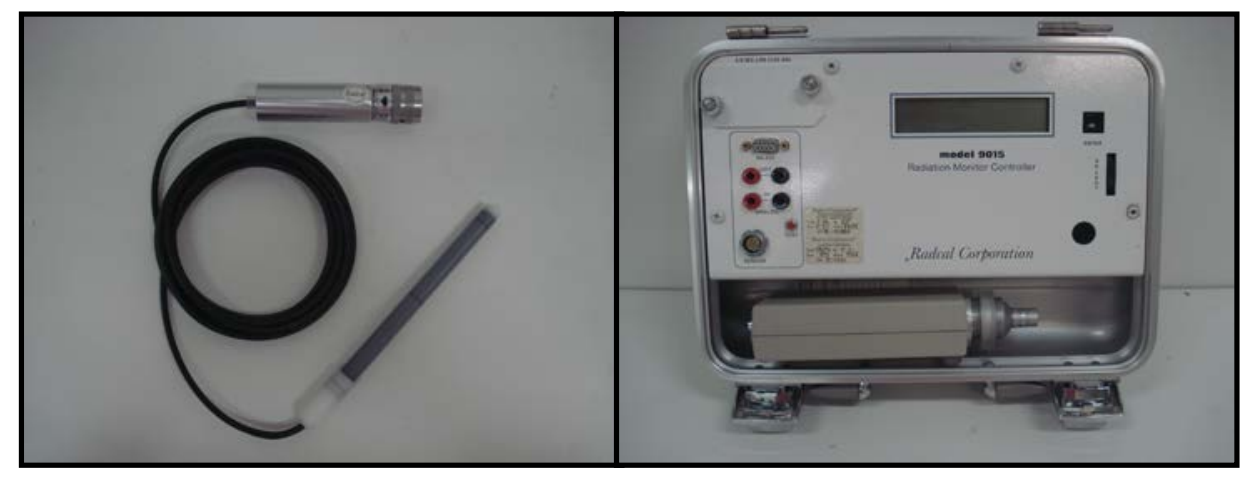

No LCI, as grandezas dosimétricas de CT foram estabelecidas em um sistema alemão de radiação X marca Pantak/Seifert, modelo MXR-160/22 para as tensões de $100 \mathrm{kV}, 120 \mathrm{kV}$ e $150 \mathrm{kV}$ (RQT 8, 9 e RQT 10), com o simulador posicionado no centro a uma distância de $100 \mathrm{~cm}$ do ponto focal, observado na Figura 2.

Figura 2: Sistema de radiação X, Pantak Isovolt HS 160 l

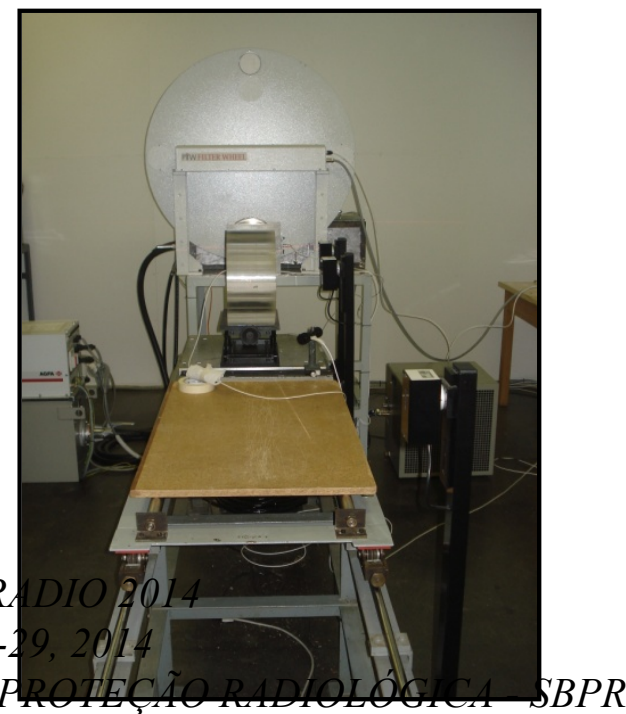


Na periferia foram realizadas as leituras com a câmara de ionização posicionada em quatro regiões denominadas: $0^{\circ}, 90^{\circ}, 180^{\circ}$ e $270^{\circ}$ como mostra a Figura 3.

Figura 3: Esquema do posicionamento da câmara de ionização em relação ao feixe de raios $X$.

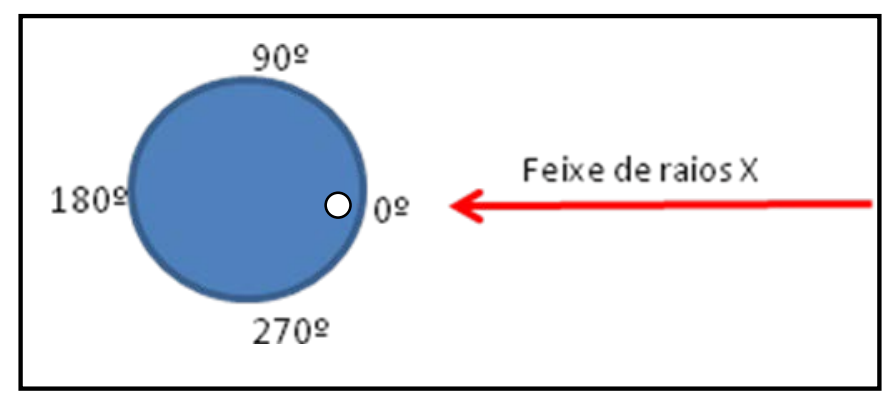

A escolha do material para a construção de um simulador físico devem ter seus materiais equivalentes a tecidos humanos, assim como o mais próximo de sua densidade e de seu coeficiente de atenuação em relação aos tecidos simulados [6].

O simulador pediátrico utilizado foi desenvolvido em quatro partes, todas em forma cilíndrica, contendo dimensões aproximadas da cabeça de uma criança de cinco anos de cinco anos de idade. Os materiais utilizados na construção obedeceram às recomendações da ICRU - Report 44 [7], como mostra a Tabela 1:

Tabela 1: Materiais de tecidos equivalentes de acordo com a ICRU - Report 44

\begin{tabular}{ccc}
\hline Materiais Substitutos & Descrição & Densidade $\left(\mathrm{g} / \mathrm{cm}^{3}\right)$ \\
\hline Acrílico (músculo) & $\begin{array}{c}\left(\mathrm{C}_{5} \mathrm{H}_{8} \mathrm{O}_{2}\right)_{\mathrm{n}} ; \\
\text { PMMA }\end{array}$ & 1,17 \\
Alumínio (osso) & $\mathrm{Al}$ & 2,7 \\
P V. C. (osso) & $\left(\mathrm{C}_{2} \mathrm{H}_{3} \mathrm{Cl}\right)_{\mathrm{n}} ;$ & 1,35 \\
\hline
\end{tabular}


Na Figura 4 podemos observar o simulador construído pelo IPEN, apresentando os quatros cilindros. O cilindro externo e o interno medem 160mm de diâmetro x $155 \mathrm{~mm}$ de altura, e 120mm de diâmetro x 155mm de altura, respectivamente. O cilindro externo contém um orifício na periferia para medições de dose de entrada na pele, já o cilindro interno contém uma válvula de escape para enchê-lo de água, quando desejado, e um orifício no centro para medições de dose absorvida. Foram construídas também duas varetas em PMMA para inserir nos orifícios com intuito de preenchimento quando não estão sendo ocupados pela câmara de ionização.

Figura 4: Simulador finalizado, parte a parte e acoplados

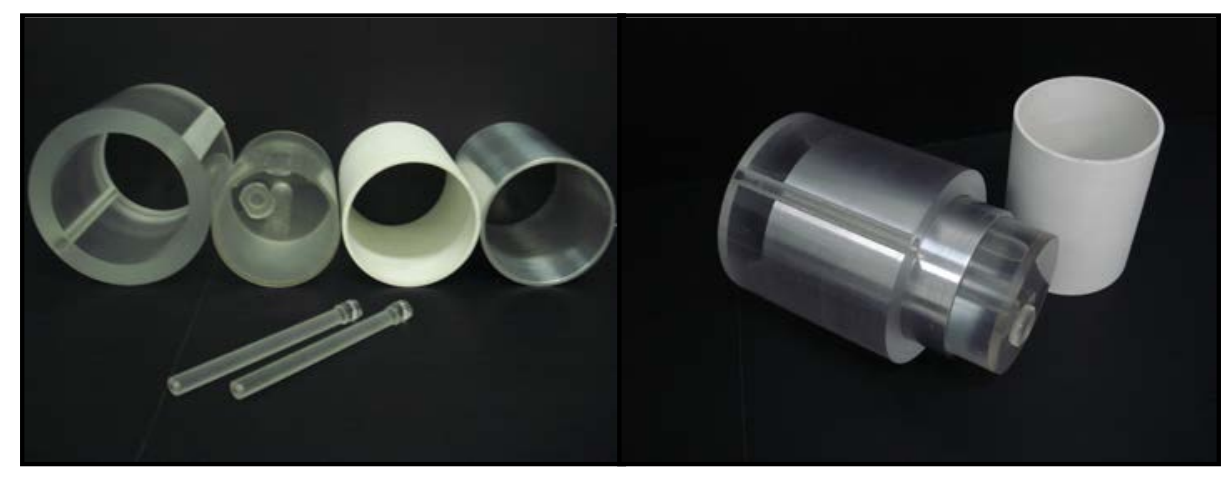

Para simular a calota craniana foram construídos dois cilindros: um de alumínio e outro de PVC ambos construídos com as mesmas dimensões 119,90mm de diâmetro externo e 111,90mm de diâmetro interno x 155mm de altura, mostrado na Figura 4. Estes são colocados entre o cilindro externo e o interno, um de cada vez.

\subsection{Medidas clínicas}

As medições clínicas foram realizadas em um tomógrafo da marca Siemens Somatom Spirit, 2 canais, com tensão de 80 a 130 kVp e corrente de 30 a 240 mA, pertencente ao Hospital Pérola Byington.

Até o momento medidas preliminares foram realizadas utilizando três cilindros: os dois construídos em PMMA (externo e interno) e o cilindro de alumínio para simular a calota craniana. Foi 
avaliado o índice de kerma no ar livre $\left(\mathrm{C}_{\mathrm{a}, 100}\right)$, e o índice ponderado de dose $\left(\mathrm{C}_{\mathrm{w}}\right)$ e foram feitas cinco leituras para cada tensão.

Para a medição do índice de kerma no ar livre $\left(C_{a, 100}\right)$, a câmara de ionização foi fixada em suporte específico de modo que a mesma se estendeu para além da extremidade da mesa. O grampo utilizado para fixar a posição da câmara de ionização em nenhum momento vetou seu volume sensível. Seu posicionamento foi realizado com o auxílio dos lasers do tomógrafo de modo que o eixo de rotação do sistema tubo-detector permita que o corte seja feito de acordo com o plano de digitalização ao volume sensível da câmara, conforme Figura 5.

Figura 5: Disposição da câmara de ionização para medição de índice de kerma no ar livre $\left(\mathrm{C}_{\mathrm{a}, 100}\right)$, inclinação $0^{\circ}$

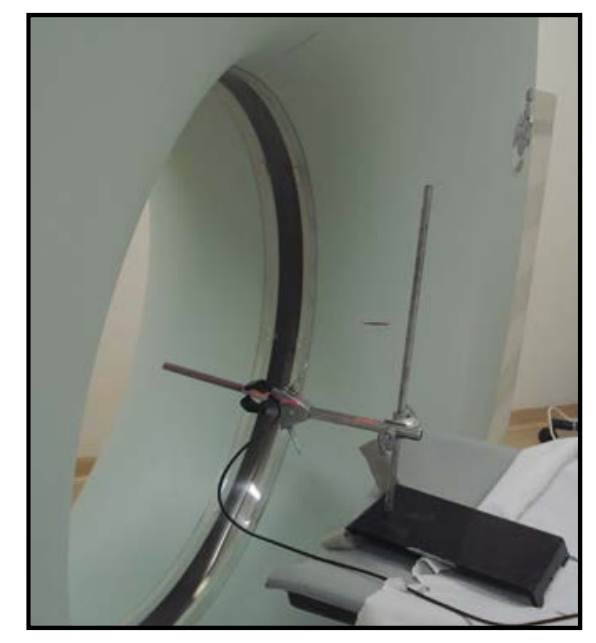

Para as medições de $\mathrm{C}_{\mathrm{PMMA}}$, 100, C e $\mathrm{C}_{\mathrm{PMMA}}$, 100, P (portanto, $\mathrm{C}_{\mathrm{W}}$ ), a câmara de ionização foi inserida no simulador e foi posicionado com o auxílio do suporte de cabeça, como mostra a Figura 6.

Figura 6: Disposição do simulador construído pelo IPEN, inclinação à $0^{\circ}$ 


\section{RESULTADOS E DISCUSSÕES}

Os índices de kerma no ar em CT: $\mathrm{C}_{\mathrm{a}, 100}$ e $\mathrm{C}_{\mathrm{w}}$ (no centro e na periferia) foram calculados de acordo com a TRS457 e os valores obtidos podem ser observados na Tabela 2. Estas medições foram realizadas no Laboratório de Calibração do IPEN com um simulador de PMMA já pertencente ao IPEN, denominado Padrão IPEN.

Tabela 2: Índices de kerma no ar em CT $\left(\mathrm{C}_{\mathrm{a}, 100}\right.$ e $\left.\mathrm{C}_{\mathrm{W}}\right)$ - valores obtidos no LCI

\begin{tabular}{ccccc}
\hline $\begin{array}{c}\text { Qualidades de } \\
\text { Radiação }\end{array}$ & $\begin{array}{c}\mathrm{C}_{\mathrm{a}, 100} \\
(\mathrm{mGy})\end{array}$ & $\begin{array}{c}\text { CPMMA,C }_{\text {PMG }} \\
(\mathrm{mG})\end{array}$ & $\begin{array}{c}\mathrm{C}_{\text {PMMA,P }} \\
(\mathrm{mGy})\end{array}$ & $\begin{array}{c}\mathrm{C}_{\mathrm{w}} \\
(\mathrm{mGy})\end{array}$ \\
\hline RQT 8 $(100 \mathrm{kV})$ & 2,267 & 2,265 & 2,047 & 2,119 \\
RQT 9 $(120 \mathrm{kV})$ & 3,480 & 3,483 & 3,141 & 3,255 \\
RQT 10 $(150 \mathrm{kV})$ & 5,770 & 5,765 & 5,188 & 5,381 \\
\hline
\end{tabular}

Os testes preliminares em feixes clínicos foram realizados no Hospital Pérola Byton, com tensão de $130 \mathrm{kV}$, utilizando os dois simuladores: o Padrão IPEN e o construído para esse trabalho. Foi calculado o índice de kerma no ar ponderado $\mathrm{C}_{\mathrm{W}}$ para os dois modelos e os valores obtidos foram apresentados na Tabela 3.

Tabela 3: Índices de kerma no ar ponderado $\left(\mathrm{C}_{\mathrm{W}}\right)$ - valores obtidos em feixes clínicos.

\begin{tabular}{cc}
\hline Simulador & Cw (mGy) \\
\hline Padrão IPEN & $3,362 \pm 0,282$ \\
Simulador desenvolvido nesse trabalho & $2,525 \pm 0,212$ \\
\hline
\end{tabular}




\section{CONCLUSÕES}

Mediante os resultados apresentados, não há dúvidas que o simulador que foi desenvolvido está adequado para procedimentos dosimétricos em avaliações das grandezas específicas em tomografia computadorizada. Os valores de incertezas de 8,4\% para feixes laboratoriais e de 14,4\% para feixes clínicos sugeridos pela TRS. $n^{\circ}$. 457 estão dentro do intervalo de confiança das medições já demonstradas no presente trabalho. No entanto, estes foram apenas testes preliminares e daremos continuidade às pesquisas de acordo com a literatura e experimentalmente.

\section{AGRADECIMENTOS}

Os autores agradecem ao Conselho Nacional de Desenvolvimento Científico e Tecnológico (CNPq), à Fundação de Amparo à Pesquisa do Estado de São Paulo (FAPESP), à Coordenação de Aperfeiçoamento de Pessoal de Nível Superior (CAPES) e ao Ministério de Ciência e Tecnologia (MCT, Projeto: Instituto Nacional de Ciência e Tecnologia (INCT) em Metrologia das Radiações na Medicina), pelo apoio financeiro parcial. 


\section{REFERÊNCIAS}

1. WHITE, K. S. Helical/spiral CT scanning: a pediatric radiology perspective, Pediatr Radiol, 26:5-14, 1996.

2. IAEA - International Atomic Energy Agency. Dosimetry in diagnostic radiology: an international code of practice. Technical Reports Series $\mathbf{N}^{\circ}$ 457, Vienna, IAEA, 2007.

3. IAEA - International Atomic Energy Agency. Implementation of the international code of practice on dosimetry in diagnostic radiology. (Technical reports series $n^{0} 457$ ), Vienna, IAEA, 2011.

4. IAEA - International Atomic Energy Agency. Dosimetry in Diagnostic Radiology for Paediatric Patients. IAEA Human Health Series no 24, Vienna, IAEA, 2013.

5. MINISTÉRIO DA SAÚDE. Diretrizes de proteção radiológica em radioadignóstico médico e odontológico, Portaria 453, Brasília, 1998.

6. HINTENLANG, D. E.; MOLONEY, W. E.; WINSLOW, J. Physical phantoms for experimental radiation dosimetry. Handbook of Anatomical Models for Radiation Dosimetry. Taylor \& Francis, 2010.

7. ICRU - International Commission on Radiation Units and Measurements. Tissue substitutes in radiation dosimetry and measurement. ICRU Report 44. U.S.A., ICRU, 1989. 